\title{
Erratum to: Secretome of tumor-associated leukocytes augment epithelial-mesenchymal transition in positive lymph node breast cancer patients via activation of EGFR/Tyr845 and NF-kB/p65 signaling pathway
}

\author{
Eslam A. El-Ghonaimy ${ }^{1}$ - Sherif A. Ibrahim ${ }^{1} \cdot$ Amal Youns $^{1} \cdot$ Zeinab Hussein $^{1}$ • \\ Mohamed Akram Nouh $^{2}$ - Tahani El-mamlouk ${ }^{1}$. Mohamed El-Shinawi ${ }^{3}$. \\ Mona Mostafa Mohamed ${ }^{1}$
}

Published online: 29 July 2016

(C) International Society of Oncology and BioMarkers (ISOBM) 2016

Erratum to: Tumor Biol.

DOI 10.1007/s13277-016-5123-x

Unfortunately, the name of the corresponding author in PubMed and the name of the first author in the published online paper were incorrectly captured.

The corresponding author publication name should have been Mohamed MM not Mostafa Mohamed M. and the name of the first author should have been Eslam A. El-Ghonaimy not Eslam A. Elghonaimy.

The online version of the original article can be found at http://dx.doi.org/ 10.1007/s13277-016-5123-x.

Mona Mostafa Mohamed mmostafa@ sci.cu.edu.eg

Eslam A. El-Ghonaimy

islamelghonaimy@yahoo.com

Sherif A. Ibrahim

isherif@sci.cu.edu.eg

Amal Youns

mollyyemira@gmail.com

Zeinab Hussein

zeinab_hk@hotmail.com

Mohamed Akram Nouh

akram.nouh@nci.cu.edu.eg
Tahani El-mamlouk

tahanielmamlouk@yahoo.com

Mohamed El-Shinawi

mohamedshinawi@med.asu.edu.eg

Department of Zoology, Faculty of Science, Cairo University, Giza 12613, Egypt

2 Department of Pathology, National Cancer Institute, Cairo University, Giza 12613, Egypt

3 Department of General Surgery, Faculty of Medicine, Ain Shams University, Cairo 11566, Egypt 